\title{
DESENVOLVIMENTO E ACEITABILIDADE DE COOKIES FORMULADOS A PARTIR DE BAGAÇO DE MANDIOCA
}

\author{
João Victor Chiquito de Carvalho ${ }^{*}$ \\ Luana Mariani Jorge ${ }^{1}$ \\ Janiely Dorini de Freitas ${ }^{1}$ \\ Marcela Moreira Terhaag ${ }^{1,2}$
}

CARVALHO, J. V. C. de; JORGE, L. M.; FREITAS, J. D. de; TERHAAG, M. M. Desenvolvimento e aceitabilidade de cookies formulados a partir de bagaço de mandioca. Arq. Cienc. Saúde UNIPAR, Umuarama, v. 21, n. 3, p, 193-199, set./dez. 2017.

RESUMO: O bagaço de mandioca, resíduo da extração de amido, apresenta alto teor de fibras e pode ser utilizado em diversas aplicações, dentre elas biscoitos. Neste trabalho objetivou-se utilizar bagaço de mandioca na produção de cookies. O bagaço foi obtido junto à empresa Cia Lorenz, da cidade de Umuarama-PR, com umidade de 84,21\% e 11,20\% de amido. As quatro formulações de cookie foram preparadas (F1; F2; F3; F4) empregando variações na proporção farinha de trigo/ bagaço de mandioca $(50 / 00 ; 27,8 / 40 ; 17,8 / 50 ; 7,8 / 60)$. Os ingredientes foram misturados em batedeira, divididos em porções, laminados na espessura média de $5 \mathrm{~mm}$ e cortados em matriz de 30 $\mathrm{mm}$ de diâmetro. Os biscoitos foram analisados antes do forneamento (AF) e após o forneamento (DF) quanto: a) ao volume de expansão e b) umidade. Os biscoitos tipo cookies foram avaliados por 100 participantes em relação a aceitação sensorial por atributos e intenção de compra. Avaliou-se o teor de fibra bruta do bagaço de mandioca e dos biscoitos formulados. A umidade (AF/DF) dos biscoitos foi de F1: 28,05\%/3,33\%; F2: 40,24\%/3,77\%; F3: 45,19\%/4,98\% e F4:54,12\%/6,17\%. O volume de expansão determinado foi de $F 1: 4,05 \mathrm{~cm}^{3} ; \mathrm{F} 2$ : $0,77 \mathrm{~cm}^{3} ; \mathrm{F} 3:-0,39 \mathrm{~cm}^{3} ; \mathrm{F} 4:-1,13 \mathrm{~cm}^{3}$. O teor de fibras brutas do bagaço foi de quase $17 \%$ e dos biscoitos foi de F1: 1, 14\%; F2: 5,51\%; F3: 7,98\% e F4: 10,22\%, demonstrando quantidade considerável de fibras nos biscoitos acrescidos do bagaço. As amostras F3 e F4 obtiveram altos escores na aceitação global (7,1 e 7,25, respectivamente) e textura (6,07 e 6,13, respectivamente). O maior nível de adição do bagaço de mandioca não proporcionou alteração na cor, aroma e sabor avaliados sensorialmente em relação a amostra isenta de bagaço. Com relação à intenção de compra, as amostras F1, F3 e F4 obtiveram as maiores médias, mostrando que há um potencial mercado para o produto proposto.

PALAVRAS-CHAVE: Avaliação sensorial. Biscoito. Fibras.

\section{DEVELOPMENT AND ACCEPTABILITY OF CASSAVA BAGASSE COOKIES}

\begin{abstract}
Cassava bagasse, the residue of starch extraction, has high fiber content and can be used in various applications, including biscuits. This study aimed at using cassava bagasse in the production of cookies. Bagasse was obtained from Cia Lorenz (Umuarama-PR), with moisture of $84.21 \pm 0.04 \%$ and $11.20 \pm 0.05 \%$ starch. Four cookie formulations were prepared (F1; F2; F3; F4) employing variations in the proportion of wheat flour/cassava bagasse $(50 / 00,27.8 / 40,17.8 / 50,7.8 / 60)$. The ingredients were mixed in the mixer, and divided into portions, laminated at an average thickness of $5 \mathrm{~mm}$ and cut in a $30-\mathrm{mm}$ diameter matrix. The biscuits were analyzed before and after baking in relation to volume expansion and moisture. Cookies were evaluated by 100 participants in relation to sensory acceptance by attributes and purchase intention. Crude fiber content of the cassava bagasse and the formulated biscuits were evaluated. Moisture content (FA/DF) of the cookies was F1: 28.05\%/3.33\%; F2: 40.24\%/3.77\%; F3: 45.19\%/4.98\% and F4: 54.12\%/6.17\%. The expansion volume was F1: $4.05 \mathrm{~cm}^{3} ; \mathrm{F} 2: 0.77 \mathrm{~cm}^{3} ; \mathrm{F} 3:-0.39 \mathrm{~cm}^{3} ; \mathrm{F} 4:-1.13 \mathrm{~cm}^{3}$. Crude fiber content of the bagasse was almost $17 \%$ and of the biscuits was F1: 1.14\%; F2: 5.51\%; F3: 7.98\% and F4: 10.22\%, demonstrating a considerable amount of fiber in the biscuits added to the bagasse. Samples F3 and F4 obtained high scores in global acceptance (7.1 and 7.25, respectively) and texture (6.07 and 6.13, respectively). The higher level of cassava bagasse did not change the color, aroma and taste evaluated in relation to the bagasse-free sample. Regarding the purchase intention, samples F1, F3 and F4 obtained the highest averages, showing that there is a potential market for the proposed product.
\end{abstract}

KEYWORDS: Biscuit. Fibers. Sensory evaluation.

\section{Introdução}

A mandioca (Manihot esculenta, Crantz) é uma planta bastante cultivada no Brasil, sendo explorada comercialmente para diversos fins, dentre os quais a extração de amido ou fécula (SARMENTO et al., 1999). O farelo, massa ou bagaço de mandioca, é o resíduo sólido da etapa de extração do amido e caracterizado como material fibroso da raiz e contendo parte da fécula que não foi possível extrair no processamento (LEONEL; CEREDA, 2000).

Biscoitos são produtos de panificação de grande interesse comercial dado as suas características de produção, comercialização e consumo, demanda elevada, relativamente longa vida de prateleira e boa aceitação, particularmente entre crianças (MORAES et al., 2010, FASOLIN et al., 2007). Muitos destes produtos têm sido desenvolvidos com a finalidade de incrementar o valor nutricional, principalmente, em relação ao teor de fibras e proteínas, visando grupos populacionais preocupados com o consumo de alimentos mais saudáveis (JAEKEL et al., 2015). Bernaud e Rodrigues (2013) citam estudos sobre o consumo adequado de fibras e a possível prevenção de doença arterial coronariana, acidente vascular cerebral, hipertensão arterial, diabetes melito e algumas desordens gastrointestinais. Há evidências de uma relação entre o consumo de fibras insolúveis e a prevenção da obesidade e síndrome metabólica (MELLO; LAAKSONEN, 2009).

Porém, esta adição de ingredientes ricos em fibras pode levar a mudanças sensoriais e tecnológicas (CLERICI et al., 2013). Inúmeros estudos foram realizados com objetivo de aproveitar o bagaço da mandioca, na formulação de rações para bovinos, como substrato para produção de etanol,

DOI: 10.25110 /arqsaude.v21i3.2017.5833

${ }^{1}$ Instituto Federal do Paraná, Campus Umuarama, Rod. PR 323 - KM 310. Pq. Industrial, CEP 87507-014, Umuarama-PR;

${ }^{2}$ Departamento de Ciência e Tecnologia de Alimentos, Universidade Estadual de Londrina, Rod. Celso Garcia Cid, PR 445, Km 380, CEP: 86055-900, Londrina/PR.*Autor para correspondência: E-mail: joaovictorcc@outlook.com 
enchimento para comprimidos, embalagens biodegradáveis e como base para produto dietético rico em fibras (CEREDA, 2001).

Atualmente, o Brasil é o segundo colocado mundial na produção de biscoitos (SEBRAE, 2014) e a adição de fibras tem sido uma alternativa tecnológica aos produtos de mercado. Na literatura existem relatos de elaboração de cookies com redução de gordura (ZAMBRANO et al., 2002), adição de farinha de aveia (GUTKOSKI et al., 2007), farinha de soja e farelo de aveia (MARETI; GROSSMANN; BENASSI, 2010), farelo de trigo e arroz (FEDDERN et al., 2011), grãos de leguminosas (ZUCCO et al., 2011), farinha integral de cevada (GUPTA; BAWA; ABU-GHANNAM; 2011) e farinha de banana verde (FASOLIN et al., 2007), sendo que os produtos apresentaram boa qualidade sensorial e tecnológica. Algumas formulações de cookies apresentam conteúdo entre 30 e $60 \%$ de lipídios, 30 e $75 \%$ de açúcar e possuem baixo teor de umidade variando entre 7 e 20\% (MORAES et al., 2010; MARETI; GROSSMANN; BENASSI, 2010).

Uma possibilidade de incremento na agroindustrialização da mandioca é o emprego de seus subprodutos industriais, como o bagaço da mandioca, que possui amido residual e volume de geração considerável no Noroeste do Paraná, em produtos de elevado valor agregado, como um cookie. Dessa forma, este trabalho objetiva avaliar o efeito da substituição de parte da farinha de trigo por bagaço de mandioca proveniente de industrias de fabricação de polvilho da região de Umuarama/PR visando produção de cookies.

\section{Métodos e Materiais}

O bagaço de mandioca $(11,20 \pm 0,05 \%$ de amido e $14,79 \pm 0,06 \%$ de carboidratos totais (IAL, 2008), conforme estudo preliminar), foi obtido junto à empresa Cia Lorenz Ltda, da cidade de Umuarama-PR, com determinação de umidade do bagaço de mandioca imediatamente após o recebimento das amostras. Para a formulação dos cookies, o bagaço de mandioca foi congelado em congelador convencional (Electrolux, Brasil) e armazenado em pacotes plásticos de PEBD até o momento da utilização. As demais matérias-primas empregadas na formulação foram obtidas no comércio local da cidade de Umuarama-PR.

\section{Método de preparo dos biscoitos tipo cookie}

As formulações de cookie (Tabela 1) foram preparadas segundo Moraes et al. (2010) com ajuste hídrico em função do teor de umidade do bagaço, sendo preparadas 4 formulações de biscoitos (F1, F2, F3, F4), empregando variações na proporção farinha de trigo/ bagaço de mandioca. As formulações foram realizadas em duplicata.

A massa dos cookies foi processada em batedeira elétrica, modelo Deluxe SX80 (Arno, Brasil), com os ingredientes secos e o bagaço de mandioca misturados por três minutos em baixa velocidade, seguida da adição de água e mistura da massa por um minuto na velocidade baixa e um minuto na velocidade média. Após a adição de toda a farinha, a massa foi misturada por dois minutos na velocidade baixa e dividida em porções, laminada na espessura média de $5 \mathrm{~mm}$ e cortada em matriz de $30 \mathrm{~mm}$ de diâmetro. Os discos de massa, com circunferência uniforme foram pesados e medidos os diâmetros antes do forneamento a $160{ }^{\circ} \mathrm{C}$ por 25 minutos, em forno elétrico com circulação de ar. Após uma hora de resfriamento, foram realizadas as análises físicas dos biscoitos sendo os restantes, armazenados em recipientes fechados hermeticamente e alocados em local fresco, seco e isento de luz até o momento da avaliação sensorial.

Tabela 1: Formulações dos biscoitos tipo cookie empregados no experimento

\begin{tabular}{l|c|c|c|c}
\hline \multirow{2}{*}{\multicolumn{1}{c}{ Ingrediente (em gramas) }} & \multicolumn{4}{c}{ Formulação } \\
\cline { 2 - 5 } & F1 & F2 & F3 & F4 \\
\hline Açúcar refinado & 20 & 20 & 17,8 & 20 \\
\hline Farinha de trigo & 50 & 27,8 & 1,3 & 7,8 \\
\hline Fermento químico & 1,3 & 1,3 & 50 & 1,3 \\
\hline Bagaço de mandioca & 0 & 40 & 0,5 & 60 \\
\hline Cloreto de sódio & 0,5 & 0,5 & 0,2 & 0,5 \\
\hline Emulsificante & 0,2 & 0,2 & 10 & 0,2 \\
\hline Margarina comum & 10 & 10 & q.s.p.* & 10 \\
\hline Água potável & 17,8 & q.s.p* & 0,2 & q.s.p.* \\
\hline Aroma de baunilha & 0,2 & 0,2 & 100 & 0,2 \\
\hline TOTAL & 100 & 100 & & 100 \\
\hline * & & & & \\
\hline
\end{tabular}

*q.s.p.: quantidade suficiente para

\section{Avaliações físico-químicas}

Os biscoitos foram analisados antes e após o forneamento quanto ao seu volume de expansão (MORAES et al., 2010), umidade e fibra bruta (IAL, 2008).

O diâmetro dos biscoitos foi determinado com ré- gua de escala milimetrada e a espessura foi determinada com paquímetro, sendo ambos mensurados antes e após o forneamento, com resultados expressos em milímetros. Essas dimensões foram empregadas para cálculo do volume específico antes e após o forneamento, conforme descrito por Mauro, Silva e Freitas (2010). Todas as análises foram realizadas em 
triplicata. Os resultados foram avaliados por análise de variância (ANOVA) de um fator, considerando-se como causa de variação o produto e testes de médias (Tukey, ao nível de 5\% de significância).

\section{Aceitabilidade sensorial}

Os biscoitos formulados foram avaliados por 100 participantes em relação à aceitação sensorial e intenção de compra, conforme projeto tramitado pelo comitê de ética (CAEE: 31471414.6.0000.0109).

Os testes sensoriais foram realizados no Instituto Federal do Paraná, Campus Umuarama, em sala adaptada para tal fim, sob luz branca. As amostras de biscoitos tipo cookies foram servidas à temperatura ambiente codificados com três dígitos (SILVA et al., 2007). Foram empregados 100 provadores que avaliaram as amostras em 1 sessão (4 amostras por sessão). A ordem e apresentação das amostras para cada provador foi aleatorizada. Foi servida água mineral à temperatura ambiente para que os provadores pudessem efetuar a limpeza do palato, entre uma amostra e outra. Foi entregue aos provadores que participaram dos testes de aceitação, uma solicitação de consentimento (ANEXO 1) para que cada provador estivesse informado do produto a ser provado.

Os provadores avaliaram as amostras com relação a cor, aroma, sabor, textura e aceitação global empregando-se escala hibrida de dez centrímetros, conforme metodologia descrita por Villanueva, Petenate e Silva (2005) (Figura 1). Foi solicitado, ainda, aos provadores que especificassem as características mais e menos apreciadas em cada amostra e que indicassem qual a intenção de compra de cada uma das amostras apresentadas (Figura 2).

Figura 1: Ficha empregada para a verificação da aceitação das amostras de biscoito tipo cookie

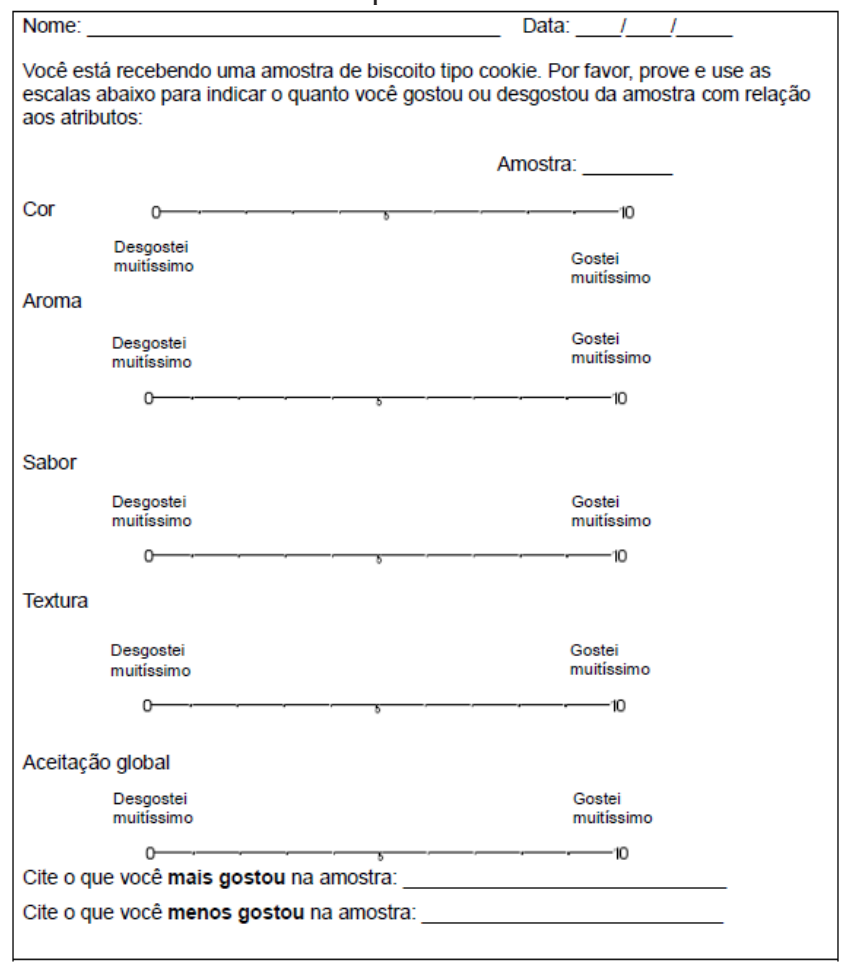

Os dados referentes a aceitação sensorial e intenção de compra foram submetidos à análise de variância (ANO-
VA), considerando-se como causas de variação amostras e provadores e teste de médias (Tukey, $\mathrm{p} \leq 0,05$ ), utilizando-se o programa Statistica 10.0 (STATSOFT, 2014).

Figura 2: Ficha empregada na verificação da intenção de compra das amostras formuladas

Considere que você precisa de biscoitos na sua casa e está por comprá-lo. A
cada amostra experimentada, anote o número da amostra e marque na
escala sua intenção de compra para o produto.

Intenção de compra:
7 - definitivamente compraria
$6-$
$5-$
$4-$
$3-$
$2-$
$1-$ definitivamente não compraria

\section{Resultados e Discussão}

\section{Análises físicas dos biscoitos}

O bagaço de mandioca imediatamente após a coleta apresentou umidade de $84,21 \pm 0,04 \%$. Esses dados são semelhantes aos obtidos por Castiglioni et al. (2013) que foi de $82,77 \%$ e também aos encontrados por Cereda (2001), $85,00 \%$ demonstrando que o resíduo fibroso absorve parte da água utilizada no processo industrial de extração do amido. O bagaço de mandioca apresentou 16,98 2 2,17\% de fibra bruta resultado semelhante $(16,08 \%)$ ao divulgado pela Embrapa (2014), indicando que há um teor considerável de fibra neste ingrediente. A Tabela 2 apresenta os resultados das determinações da umidade e volume de expansão dos biscoitos tipo cookies antes e após o forneamento.

De acordo com os teores de umidades da massa verificou-se que as formulações propostas diferiram entre si. Verificou-se maiores teores de umidade antes do forneamento nas amostras que possuíam maiores teores de bagaço de mandioca, já que este é um ingrediente com $84,21 \pm 0,04 \%$ de água. Após o forneamento verificou-se que as amostras F3 e F4 diferiram da amostra padrão (F1) que não continha bagaço de mandioca, demonstrando que parte da água contida no bagaço ficou retida pela massa durante o processo de produção do biscoito. Segundo resolução da Anvisa (1978) a umidade dos biscoitos deve ser no máximo de $14,00 \%$ e observamos que os biscoitos formulados apresentaram teores de umidade dentro dos padrões da legislação vigente. 
Tabela 2: Dados de umidade antes e após forneamento e expansão dos biscoitos tipo cookies formulados ${ }^{(1)}$

\begin{tabular}{c|c|c|c|c}
\hline \multirow{2}{*}{ Formulação } & \multirow{2}{*}{ Volume de expansão $\left(\mathbf{c m}^{3}\right)$} & \multicolumn{2}{|c|}{ Umidade (\%) } & \multirow{2}{*}{ Fibra bruta (\%) } \\
\cline { 3 - 5 } & & Antes do forneamento & Após forneamento & \\
\hline F1 & $4,05 \pm 0,05 \mathrm{a}$ & $28,05 \pm 0,08 \mathrm{~d}$ & $3,33 \pm 0,62 \mathrm{c}$ & $1,14 \pm 0,29 \mathrm{c}$ \\
\hline F2 & $0,77 \pm 0,12 \mathrm{~b}$ & $40,24 \pm 0,05 \mathrm{c}$ & $3,77 \pm 0,73 \mathrm{bc}$ & $5,51 \pm 1,52 \mathrm{bc}$ \\
\hline F3 & $-0,39 \pm 0,06 \mathrm{c}$ & $45,19 \pm 0,04 \mathrm{~b}$ & $4,98 \pm 0,72 \mathrm{ab}$ & $7,98 \pm 0,73 \mathrm{ab}$ \\
\hline F4 & $-1,13 \pm 0,16 \mathrm{~d}$ & $54,12 \pm 0,30 \mathrm{a}$ & $6,17 \pm 0,11 \mathrm{a}$ & $10,22 \pm 1,51 \mathrm{a}$ \\
\hline
\end{tabular}

(1) Resultados expressos como média \pm desvio padrão; média seguidas de letras distintas na mesma coluna indicam diferença significativa a $\mathrm{p} \leq 0,05(\mathrm{n}=8)$.

Segundo Mareti et al. (2010), na substituição parcial da farinha de trigo por fontes proteicas e de fibras, é possível que ocorram problemas tecnológicos, provocadas pelo acrescimento de grupos hidrofílicos, sendo indicado o acréscimo de água na formulação, para que a massa não fique seca e quebradiça. Os ingredientes usados na elaboração de biscoitos afetam grandemente a sua qualidade (MORAES et al., 2010) sendo que a farinha de trigo contribui na qualidade global. No caso da substituição parcial da farinha de trigo por bagaço de mandioca não foi necessário o acréscimo de água na formulação, pois o bagaço de mandioca, fonte de fibras, já contém aproximadamente $84,21 \%$ de água em sua constituição. Estes dados são semelhantes aos obtidos por Ramos et al, (2000) que foi de 82,77\% (CASTIGLIONI et al., 2013) e também aos encontrados por Cereda (2001), que obteve $85 \%$.

Também são afetadas as propriedades de mistura da massa (tempo de desenvolvimento, estabilidade, tolerância à mistura), sua elasticidade e extensibilidade e as características do produto final (volume, cor, umidade e dureza) (SUDHA et al., 2007 apud MARETI et al., 2010). Foi possível perceber uma alteração na textura e no volume de expansão dos biscoitos produzidos com a substituição parcial da farinha de trigo pelo bagaço de mandioca. Tais conclusões podem ser visualizadas nos resultados das análises de volume de expansão e umidade que foram feitas das formulações desenvolvidas.

Verificou-se que dentre as amostras formuladas, que a adição do bagaço de mandioca interferiu negativamente no volume de expansão das amostras, sendo que os biscoitos contendo este ingrediente apresentaram menor crescimento em relação ao padrão (F1), sendo inclusive negativo para as amostras (F3 e F4) que continham 50 e $60 \%$ de bagaço, respectivamente, explicando a influência negativa do bagaço na expansão do biscoito durante o forneamento. Isso pode ser relacionado ao menor teor de farinha de trigo nas amostras F3 e F4. Possivelmente, essa menor expansão está relacionada ao menor teor de farinha de trigo adicionada às formulações F2, F3 e F4 e a consequente menor capacidade destas de aprisionar o $\mathrm{CO}_{2}$ liberado pelo fermento químico durante o forneamento, relacionado ao menor teor de glúten nas formulações F2, F3 e F4.

Silva et al. (2001) obtiveram um maior diâmetro e uma menor espessura na formulação padrão em comparação a formulações adicionadas de farinha de jatobá (Hymenaea stigonocarpa Mart.) e cita que essa diferença pode ter ocorrido devido a maior higroscopicidade dos materiais fibrosos presentes na farinha de jatobá, que retêm a água, dando maior consistência à massa, evitando seu espalhamento, o que geralmente é a causa do maior diâmetro e menor espes- sura. Isso confirma o observado nas formulações de biscoito contendo bagaço de mandioca, já que as formulações contendo maiores teores de bagaço apresentaram menores taxas de expansão.

Fasolin et al. (2007) em biscoitos produzidos empregando farinha de banana verde verificou que maiores teores de farinha de banana verde (FBV) tende a diminuir a espessura e aumentar o diâmetro com o aumento da porcentagem de FBV, possivelmente pela ausência de glúten na FBV. Os resultados da expansão das amostras tiveram grande contraste em relação outras pesquisas similares. Biscoitos produzidos com farinha de banana (FASOLIN, 2007) apresentaram

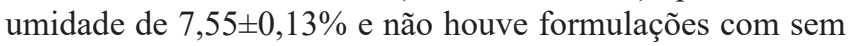
crescimento ou que encolheram. Já amostras de cookies confeccionados com farinha de talo de couve (FTC) e farinha de talo de espinafre (FTE) (MAURO; SILVA; FREITAS, 2010) foram caracterizados com (FTC) $5,80 \pm 0,45 \%$ de umidade e (FTE) $4,74 \pm 0,09 \%$. Também não houve crescimento retrógrado das formulações, demonstrando que a adição de material fibroso manteve os biscoitos da dimensão de modelagem após o forneamento.

O bagaço de mandioca proveniente da extração industrial do amido, da região de Umuarama/PR, apresentou um teor de fibras brutas de $16,98 \pm 2,17 \%$, o resultado foi semelhante ao divulgado pela Embrapa (2014). Em relação ao teor de fibras dos biscoitos formulados, verificou-se que as amostras contendo bagaço de mandioca apresentaram teores entre 5,51 e 10,22\%.

Os biscoitos F4 e F3 apresentaram maiores teores de fibra em relação ao padrão (F1), o que corrobora com a formulação proposta, já que os biscoitos $\mathrm{F} 3$ e $\mathrm{F} 4$ possuem maiores proporções de bagaço de mandioca. De acordo com Anvisa (2016) caso o alimento forneça mais de 2,5g de fibras por porção o mesmo pode utilizar uma alegação de rotulagem de que "as fibras alimentares auxiliam o funcionamento do intestino" e "seu consumo deve estar associado a uma alimentação equilibrada e hábitos de vida saudáveis". Dessa forma, a ingestão dos biscoitos tipo cookies adicionados de bagaço de mandioca (F4) associada a uma alimentação saudável pode auxiliar no funcionamento intestinal e uma alternativa de produto funcional. A adição das fibras do bagaço de mandioca nos biscoitos tipo cookies pode proporcionar um valorização deste subproduto da agroindústria brasileira além de proporcionar um alimento nutricionalmente mais adequado.

Os resíduos de alimentos utilizados na indústria alimentícia são geralmente, desprezados e poderiam ser utilizados como fonte alternativa de fibras (MAURO; SILVA; FREITAS, 2010) adicionados a diversos produtos, inclusive para alimentação escolar. Quando comparado o teor de fibras 
determinados em outros cookies formulados, biscoitos contendo farinha de aveia e de passas (VOLLENDORF E MARLETT, 1994) obtiveram valores de 2,7 a 4,3\% (em base úmida), Perez e Germani (2007) encontraram valores de 8,22\% (em base seca) em cookies confeccionados com 15\% de farinha de berinjela, Silva (2001) encontrou níveis de 4,1 a 6,5\% de fibra alimentar total (em base seca) em cookies elaborados com farinha mista de trigo e jatobá. Protzek (1998) elaborou biscoitos com diferentes níveis de substituição de farinha de trigo por farinha de bagaço de maçã, encontrando valores de fibra alimentar total de 2,70 a 6,05\%. Clerici et al. (2013) em pesquisa com biscoitos tipo cookies com farinha desengordurada de gergelim determinou fibra alimentar (3,07\%). Isso demonstra que os cookies contendo bagaço de mandioca foram formulados contendo maior teor de fibras que os pes- quisados, o que pode ter interferido nos parâmetros físicos de expansão e umidade do produto forneado.

\section{Aceitabilidade e intenção de compra dos biscoitos tipo cookie}

Os biscoitos foram avaliados sensorialmente por 100 provadores, sendo a média das avaliações sensoriais apresentadas na Tabela 3. Conforme dados, verificou-se que as amostras não apresentaram diferença significativa $(\mathrm{p} \leq 0,05)$ com relação a cor, o que demonstra que a substituição da farinha de trigo por bagaço não influenciou na cor do produto. Protzek et al. (1998) encontrou resultado semelhante quando avaliou sensorialmente biscoitos produzidos com bagaço de maçã.

Tabela 3: Aceitação sensorial dos cookies de bagaço de mandioca ${ }^{(1)}$

\begin{tabular}{c|c|c|c|c|c}
\hline Amostra & Cor & Aroma & Sabor & Textura & Aceit. Global \\
\hline F1 & $7,68 \pm 1,84^{\mathrm{a}}$ & $7,50 \pm 2,01^{\mathrm{a}}$ & $8,08 \pm 1,85^{\mathrm{a}}$ & $6,75 \pm 2,17^{\mathrm{a}}$ & $7,87 \pm 1,81^{\mathrm{a}}$ \\
\hline F2 & $7,51 \pm 2,28^{\mathrm{a}}$ & $7,03 \pm 2,26^{\mathrm{ab}}$ & $6,72 \pm 2,66^{\mathrm{ab}}$ & $5,76 \pm 2,76^{\mathrm{b}}$ & $6,70 \pm 2,54^{\mathrm{b}}$ \\
\hline F3 & $7,34 \pm 1,94^{\mathrm{a}}$ & $6,74 \pm 2,00^{\mathrm{b}}$ & $6,87 \pm 2,39^{\mathrm{b}}$ & $6,07 \pm 2,30^{\mathrm{ab}}$ & $7,10 \pm 2,21^{\mathrm{ab}}$ \\
\hline F4 & $7,73 \pm 1,91^{\mathrm{a}}$ & $7,35 \pm 1,89^{\mathrm{ab}}$ & $7,24 \pm 2,19^{\mathrm{ab}}$ & $6,13 \pm 2,28^{\mathrm{ab}}$ & $7,25 \pm 2,29^{\mathrm{ab}}$ \\
\hline
\end{tabular}

(1) Resultados expressos como média \pm desvio padrão; média seguidas de letras distintas na mesma coluna indicam diferença significativa a $\mathrm{p} \leq 0,05$

Com relação ao aroma, as amostras F1, F2 e F4 apresentaram as maiores médias. A amostra F4, que continha bagaço de mandioca em maior proporção apresentou médias semelhantes $(\mathrm{p} \leq 0,05)$ a amostra padrão $(\mathrm{F} 1)$ em relação ao sabor e textura. Este resultado demonstra que a adição do bagaço de mandioca na proporção de $60 \%$ proporciona produto formulado biscoitos com sabor e textura com uma boa aceitação. De forma contraria, Silva et al. (2001) verificou que a adição de farinha de jatobá-do-cerrado e jatobá-da-mata em biscoitos influenciou negativamente na textura e sabor. Fasolin et al. (2007) observaram em avaliação sensorial de em biscoitos contendo farinha de banana verde, notas variando entre 7,03 a 7,13, com escala hedônica variando de 1 a 9 , com provadores adultos.

$\mathrm{Na}$ aceitação sensorial global, as amostras F1 e F2 foram consideradas diferentes pelos provadores. Já as amos- tras que continham bagaço de mandioca, F3 e F4 obtiveram altos escores e foram consideradas semelhantes ao padrão. Os resultados mostraram que o aumento da substituição da farinha de trigo por bagaço de mandioca não interferiu na aceitação global dos cookies pelos provadores. Silva et al. (2001) encontrou resultados semelhantes em biscoitos com adição de farinha de jatobá. Já Clerici et al. (2013) encontrou resultados verificou menor aceitação global em biscoitos tipo cookies elaborados com substituição parcial da farinha de trigo por farinha desengordurada de gergelim.

$\mathrm{Na}$ intenção de compra (Tabela 4), cuja escala variou de 7 a 1, dentre as amostras que continham bagaço de mandioca, as formulações F3 e F4 obtiveram as maiores médias (4,88 e 4,94, respectivamente) e mostrando que há um mercado potencial para o produto proposto.

Tabela 4: Intenção de compra das amostras de biscoitos formulados ${ }^{(1)}$

\begin{tabular}{c|c|c|c|c}
\hline Amostra & F1 & F2 & F3 & F4 \\
\hline Intenção de compra & $5,48 \pm 1,49^{\mathrm{a}}$ & $4,52 \pm 1,88^{\mathrm{b}}$ & $4,88 \pm 1,69^{\mathrm{ab}}$ & $4,94 \pm 1,70^{\mathrm{ab}}$ \\
\hline
\end{tabular}

${ }^{(1)}$ Resultados expressos como média \pm desvio padrão; média seguidas de letras distintas na mesma coluna indicam diferença significativa a $\mathrm{p} \leq 0,05$

Piovesana, Bueno e Klajn (2013) verificaram que com relação à intenção de compra, dentre os biscoitos adicionados de aveia e farinha do bagaço de uva, não houve diferença significativa nos resultados de intenção de compra. Porém, percebe-se uma leve desvantagem na nota em função do aumento da adição das fontes de fibra: farinha do bagaço de uva e aveia (PIOVESANA; BUENO; KLAJN, 2013).

Segundo métrica projetadas para 2020, pela FIESP e pela ITAL, (VIALTA et al., 2015) observa-se que há uma tendência de consumo de alimentos convenientes e práticos, preferencialmente com características de funcionalidade, sendo que esta pode ser uma justificativa para a compra de um cookie funcional adicionado de bagaço de mandioca, já que ele cumpre parte das expectativas desta fração do mercado consumidor.

\section{Conclusão}

Os biscoitos tipo cookie formulados com adição de bagaço de mandioca apresentaram uma maior umidade em relação à amostra padrão (F1). A formulação com maior teor de bagaço apresentou maior teor de fibras em relação ao padrão. A adição do bagaço de mandioca interferiu negativamente no volume de expansão das amostras sendo que nas 
concentrações testadas, quanto maior a adição de bagaço de mandioca menor o volume de expansão verificado.

Os biscoitos contendo maior proporção de bagaço de mandioca na formulação não diferiram estatisticamente em relação à amostra isenta de bagaço em relação a cor, aroma, sabor, textura e aceitação global, apresentando uma boa aceitabilidade sensorial. Com relação à intenção de compra, os biscoitos formulados com adição do bagaço de mandioca também obtiveram notas altas, mostrando que há um potencial mercado para o produto.

Dessa forma, concluímos que a adição do bagaço de mandioca nas proporções testadas, às formulações de cookie torna o produto com maior teor de fibra bruta, com boa aceitabilidade sensorial e potencial de compra, demonstrando a viabilidade do produto proposto.

\section{Referências}

AGÊNCIA NACIONAL VIGILÂNCIA SANITÁRIA ANVISA. Resolução - CNNPA nº 12, de 1978. Diário Oficial [da] República Federativa do Brasil, Brasília, DF, 24 jul. 1978.

\section{ANVISA. Alimentos com Alegacoes de Propriedades} Funcionais e ou de Saúde. 2016. Disponível em: $<$ http:// portal.anvisa.gov.br/alimentos/alegacoes $>$. Acesso em: 02 mai. 2017.

BERNAUD, F. S. R.; RODRIGUES, T. C. Fibra alimentarIngestão adequada e efeitos sobre a saúde do metabolismo. Arquivos Brasileiros de Endocrinologia e Metabologia, v. 57, n. 6, p. 397-405, 2013.

CASTIGLIONI, G. L. et al. Modelagem matemática do processo de secagem da massa fibrosa. Revista Brasileira de Engenharia Agrícola e Ambiental, v.17, n. 9, p.987994, 2013.

CEREDA, M. P. Propriedades gerais do amido. São Paulo: Fundação Cargill, 2001, v. 1, 221 p.

CLERICI, M. T. P. S.; OLIVEIRA, M. E.; NABESHIMA, E. H. Qualidade física, química e sensorial de biscoitos tipo cookies elaborados com a substituição parcial da farinha de trigo por farinha desengordurada de gergelim. Brazilian Journal of Food Technology, v. 16, n. 2, p. 139-146, 2013.

\section{EMBRAPA: EMPRESA BRASILEIRA DE PESQUISA AGROPECUÁRIA. Subprodutos da mandioca -} composição dos resíduos sólidos. Disponível em <http:// info.cnptia.embrapa.br/digital/bitstream/item/24804/1/ folder-mandioca.pdf $>$ Acesso em: 24 dez. 2014.

FASOLIN, L. H. et al. Biscoitos produzidos com farinha de banana: avaliações química, física e sensorial. Ciência e Tecnologia de Alimentos, v. 3, n. 27, p. 524-529, 2007.

FEDDERN, V. et al. Avaliação física e sensorial de biscoitos tipo cookie adicionados de farelo de trigo e arroz. Brazilian Journal of Food Technology, v. 14, n. 4, p. 267-274, 2011.
GUPTA, M.; BAWA, A. S.; ABU-GHANNAM, N. Effect of barley flour and freezethaw cycles on textural nutritional and functional properties of cookies. Food and Bioproducts Processing, v. 89, p. 520-527, 2011.

Instituto Adolfo Lutz (IAL) (2008) Métodos físicoquímicos para análise de alimentos. 4. ed. São Paulo, Instituto Adolfo Lutz. 1020p. (Versão eletrônica)

\section{JAEKEL, L. Z. et al. Caracterização físico-química e} avaliação sensorial de biscoito tipo "cookies" com grãos de soja. Disponível em: <http://www.ufpel.edu.br/cic/2004/ arquivos/CA_00785.rtf>. Acesso em: 30 jan. 2015.

LEONEL, M.; CEREDA, M. P. Extração da fécula retida no resíduo fibroso do processo de produção de fécula de mandioca. Ciência e Tecnologia de Alimentos, v. 20, n. 1, p. 122-127, 2000.

MARETI, M. C.; GROSSMANN, M. V. E.; BENASSI, M. T. Características físicas e sensoriais de biscoitos com farinha de soja e farelo de aveia. Ciência e Tecnologia de Alimentos, v. 30, n. 4, p. 878-883, 2010.

MAURO, A. K.; SILVA, V. L. M.; FREITAS, M. C. J. Caracterização física, química e sensorial de cookies confeccionados com Farinha de Talo de Couve (FTC) e Farinha de Talo de Espinafre (FTE) ricas em fibra alimentar. Ciência e Tecnologia de Alimentos, v. 30, n. 3, p. 719-728, 2010 .

MELLO, V. D.; LAAKSONEN, D. E. Fibras na dieta: tendências atuais e benefícios à saúde na síndrome metabólica e no diabetes melito tipo 2. Arquivos

Brasileiros de Endocrinologia e Metabologia, v. 53, n. 5, p. 509-518, 2009.

MORAES, K. S. et al. Avaliação tecnológica de biscoitos tipo cookie com variações nos teores de lipídio e de açúcar. Ciência e Tecnologia de Alimentos, v. 30 (Supl.1), p. 233242, 2010.

PEREZ, P. M. P.; GERMANI, R. Elaboração de biscoitos tipo salgado, com alto teor de fibra alimentar, utilizando farinha de berinjela (Solanum melongena, L.). Ciência e Tecnologia de Alimentos, v. 7, n. 1, p. 186-92, 2007.

PIOVESANA, A.; BUENO, M. M.; KLAJN, V. M. Elaboração e aceitabilidade de biscoitos enriquecidos com farinha de veia e farinha de bagaço de uva. Brazilian Jornal of Food Tecnology, v. 16, n. 1, p. 68-72, 2013.

PROTZEK, E. C.; FREITAS, R. J. S.; WASCZYNSKJ, N. Aproveitamento do bagaço de maçã na elaboração de biscoitos ricos em fibra alimentar. Boletim do CEPPA, v.16, n.2 p.264-273, 1998.

SILVA, J. B. et al. Aceitabilidade de bebidas preparadas a partir de diferentes extratos hidrossolúveis de soja. Pesquisa Agropecuária Brasileira, v. 42, n. 12, p. 11791784, 2007. 
SILVA, M. R. et al. Utilização tecnológica dos frutos de jatobá-do-cerrado e de jatobá-da-mata na elaboração de biscoitos fontes de fibra alimentar e isentos de açúcares. Ciência e Tecnologia de Alimentos, v.2, n. 21, p.176-182, 2001.

STATSOFT. Statistica for Windows [Computer program manual]. Versão 10.0. Tulsa: StatSoft. Disponível em: $<$ http://www.statsoft.com>. Acesso em: 30 jul. 2014

VIALTA, A. et al. Brasil Food Trends. Federação das Indústrias do Estado de São Paulo/ Instituto de Tecnologia de Alimentos. São Paulo, 2010. Disponível em: < http:// www.brasilfoodtrends.com.br/Brasil_Food_Trends/index. html> Acesso: 31 jan. 2015

VILLANUEVA, N. D. M.; PETENATE, A. J.; DA SILVA, M. A. A. P. Performance of hybrid hedonic scale as compared to the traditional hedonic, self-adjusting and ranking scales. Food Quality and Preference, v. 16, p. 691-703, 2005

Recebido em: 02/12/2016

Aceito em: 05/10/2017 